\title{
Periodontitis and diabetes: a two-way relationship
}

\author{
P. M. Preshaw • A. L. Alba • D. Herrera • S. Jepsen • \\ A. Konstantinidis $\cdot$ K. Makrilakis $\cdot$ R. Taylor
}

Received: 25 March 2011 / Accepted: 21 June 2011 /Published online: 6 November 2011

(C) The Author(s) 2011. This article is published with open access at Springerlink.com

\begin{abstract}
Periodontitis is a common chronic inflammatory disease characterised by destruction of the supporting structures of the teeth (the periodontal ligament and alveolar bone). It is highly prevalent (severe periodontitis affects $10-15 \%$ of adults) and has multiple negative impacts on quality of life. Epidemiological data confirm that diabetes is a major risk factor for periodontitis; susceptibility to periodontitis is
\end{abstract}

P. M. Preshaw $(\bowtie)$

School of Dental Sciences and Institute of Cellular Medicine,

Newcastle University,

Framlington Place,

Newcastle upon Tyne NE2 4BW, UK

e-mail: p.m.preshaw@ncl.ac.uk

A. L. Alba

Endocrinology Department, Hospital de Jove,

Gijon, Spain

D. Herrera

Faculty of Odontology, Complutense University,

Madrid, Spain

S. Jepsen

Department of Periodontology,

Operative and Preventive Dentistry, University of Bonn,

Bonn, Germany

A. Konstantinidis

Dental School, Aristotle University of Thessaloniki,

Thessaloniki, Greece

K. Makrilakis

Department of Internal Medicine,

University of Athens Medical School,

Athens, Greece

R. Taylor

School of Medical Sciences and Institute of Cellular Medicine,

Newcastle University,

Newcastle upon Tyne, UK increased by approximately threefold in people with diabetes. There is a clear relationship between degree of hyperglycaemia and severity of periodontitis. The mechanisms that underpin the links between these two conditions are not completely understood, but involve aspects of immune functioning, neutrophil activity, and cytokine biology. There is emerging evidence to support the existence of a two-way relationship between diabetes and periodontitis, with diabetes increasing the risk for periodontitis, and periodontal inflammation negatively affecting glycaemic control. Incidences of macroalbuminuria and end-stage renal disease are increased twofold and threefold, respectively, in diabetic individuals who also have severe periodontitis compared to diabetic individuals without severe periodontitis. Furthermore, the risk of cardiorenal mortality (ischaemic heart disease and diabetic nephropathy combined) is three times higher in diabetic people with severe periodontitis than in diabetic people without severe periodontitis. Treatment of periodontitis is associated with $\mathrm{HbA}_{1 \mathrm{c}}$ reductions of approximately $0.4 \%$. Oral and periodontal health should be promoted as integral components of diabetes management.

Keywords Diabetes - Diabetes complications · Periodontal diseases · Periodontitis · Type 1 diabetes mellitus - Type 2 diabetes mellitus

$\begin{array}{ll}\text { Abbreviations } \\ \text { CRP } & \text { C-reactive protein } \\ \text { ESRD } & \text { End-stage renal disease } \\ \text { GCF } & \text { Gingival crevicular fluid } \\ \text { INVEST } & \text { Oral Infections and Vascular Disease } \\ & \text { Epidemiology Study } \\ \text { MMP } & \text { Matrix metalloproteinase } \\ \text { NHANES } & \text { National Health and Nutrition Examination } \\ & \text { Survey }\end{array}$




$\begin{array}{ll}\text { PGE } & \text { Prostaglandin E } \\ \text { PMN } & \text { Polymorphonuclear leucocyte } \\ \text { ROS } & \text { Reactive oxygen species }\end{array}$

\section{The two-way relationship between diabetes and periodontitis}

What is periodontitis?

Periodontal diseases are collectively the most common diseases known to mankind. Their classification is complex and takes into account the clinical presentation, age at diagnosis, rate of disease progression, and systemic and local factors that may increase risk. Periodontal diseases include gingivitis (in which the inflammation is confined to the gingiva, and is reversible with good oral hygiene) and periodontitis (in which the inflammation extends and results in tissue destruction and alveolar bone resorption). Tissue destruction in periodontitis results in breakdown of the collagen fibres of the periodontal ligament, resulting in the formation of a periodontal pocket between the gingiva and the tooth. 'Pocketing' is not evident on simple visual inspection, and assessment using a periodontal probe is essential. Periodontitis is a slowly progressing disease but the tissue destruction that occurs is largely irreversible. In the early stages, the condition is typically asymptomatic; it is not usually painful, and many patients are unaware until the condition has progressed enough to result in tooth mobility. The pockets deepen as a result of the further destruction of fibres of the periodontal ligament (referred to as attachment loss; Fig. 1) and the resorption of the alveolar bone that occurs in parallel with the progressing attachment loss. Advanced periodontitis is characterised by gingival erythema and oedema, gingival bleeding, gingival recession, tooth mobility, drifting of teeth, suppuration from periodontal pockets, and tooth loss.

The condition is very common, with severe periodontitis that threatens tooth retention affecting $10-15 \%$ of adults in most populations studied [1-3]. Moderate periodontitis is even more common, affecting $40-60 \%$ of adults. Periodontitis is therefore a highly prevalent, but largely hidden, chronic inflammatory disease. Furthermore, it has negative and profound impacts on many aspects of daily living and quality of life, affecting confidence, social interactions and food choices [4]. Smoking is a major risk factor; it significantly increases risk for periodontitis and severity of the condition $[5,6]$. Other risk factors for periodontal diseases include diabetes, conditions associated with compromised immune responses (e.g. HIV), nutritional defects, osteoporosis, medications that cause drug induced gingival overgrowth (e.g. some calcium channel blockers, phenytoin, ciclosporin), genetic factors (as yet

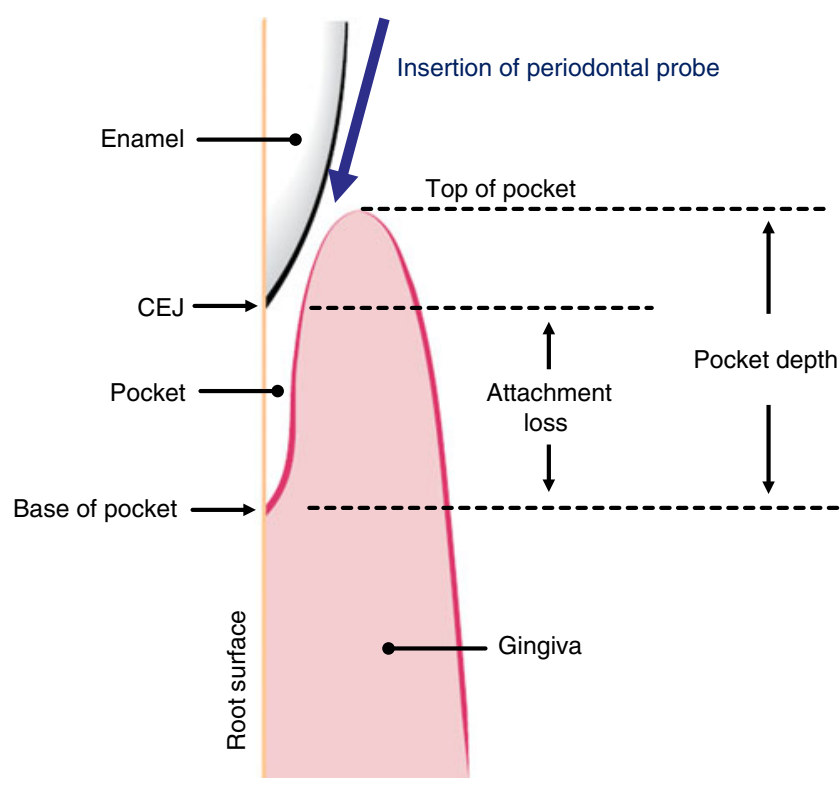

Fig. 1 Diagram of periodontal pocket in a patient with periodontitis. The pocket is the space between the root surface and the gingiva. In healthy gums, the base of the pocket is coincident with the cementoenamel junction (CEJ, the boundary between the enamel crown and the root) and there is no attachment loss. In periodontitis, the base of the pocket migrates apically (i.e. away from the enamel crown towards the root tip), thereby creating a pocket. The base of the pocket is therefore apical to the CEJ, and attachment loss can be measured (in $\mathrm{mm}$, using a periodontal probe) from the CEJ to the base of the pocket. Pocket depth (also called probing depth) is measured in $\mathrm{mm}$ from the top of the pocket (i.e. from the gingival margin) to the base of the pocket. In this example, the pocket depth might be $6 \mathrm{~mm}$, with $4 \mathrm{~mm}$ loss of attachment (as indicated in this example, pocket depth is usually greater than attachment loss due to the inflammation-induced swelling of the gingiva). The direction of insertion of a periodontal probe is indicated

poorly defined), and local factors (e.g. anatomical deficiencies in the alveolar bone) [5].

Associations between diabetes and periodontitis

Diabetes has been unequivocally confirmed as a major risk factor for periodontitis [7-9]. The risk of periodontitis is increased by approximately threefold in diabetic individuals compared with non-diabetic individuals [10]. The level of glycaemic control is of key importance in determining increased risk. For example, in the US National Health and Nutrition Examination Survey (NHANES) III, adults with an $\mathrm{HbA}_{1 \mathrm{c}}$ level of $>9 \%$ had a significantly higher prevalence of severe periodontitis than those without diabetes (OR 2.90; 95\% CI 1.40, 6.03) after controlling for age, ethnicity, education, sex and smoking [11]. The importance of diabetes as a major risk factor for periodontitis became apparent in the 1990s in a number of cross-sectional and longitudinal studies investigating the Pima Indian population. The prevalence and incidence of periodontitis were greater in Pima Indians who 
had type 2 diabetes mellitus compared with those who did not $[12,13]$, with an approximately threefold increased risk for periodontitis [14]. The majority of research has focused on type 2 diabetes mellitus as a risk factor for periodontitis, probably because both diseases have historically tended to develop in patients in their $40 \mathrm{~s}$ and 50s. However, type 1 diabetes mellitus also increases the risk of periodontitis, and all patients with diabetes (including children and young adults) should be considered to be at increased risk of periodontitis. One early study identified that around $10 \%$ of children $(<18$ years $)$ with type 1 diabetes mellitus had increased attachment loss and bone loss compared with controls, despite comparable plaque scores [15]. More recently, in a study of 350 diabetic children (618 years old) vs 350 non-diabetic controls, the proportion of periodontal sites with evidence of periodontitis was greater in the children with diabetes $(>20 \%$ vs $8 \%$ of sites, respectively) [16].

Dentists have long been aware of the importance of a diagnosis of diabetes in their patients, and various oral conditions are associated with diabetes, including xerostomia and candidal infections as well as periodontitis. In the early 1990s periodontitis was sometimes referred to as the 'sixth complication of diabetes' [17], and in 2003 the ADA acknowledged that periodontal disease is often found in people with diabetes [18]. The clinical and radiographic appearance of periodontitis is shown in Figs 2 and 3.

\section{Associations between obesity and periodontitis}

Other lifestyle factors such as obesity, physical activity and diet are also likely to affect the risk of periodontitis. One of the first studies to show an effect of obesity on periodontitis identified that obese rats with periodontitis had more

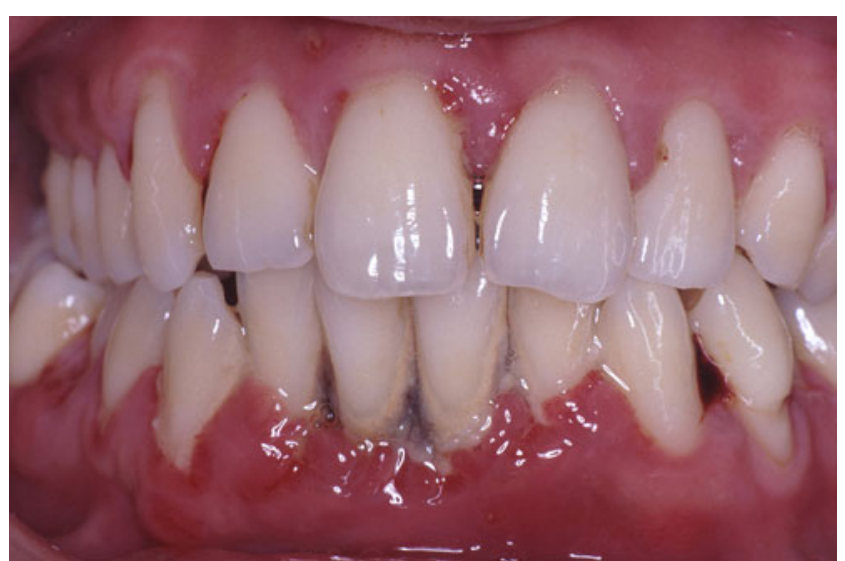

Fig. 2 Periodontitis (clinical appearance) in a 22-year-old man with poorly controlled type 1 diabetes and severe periodontitis. Note the generalised inflammation, abnormal gingival anatomy owing to tissue destruction, gingival recession, swelling and inflammation, spontaneous bleeding and abundant plaque deposits. The periodontal tissues around the lower incisors are particularly severely affected
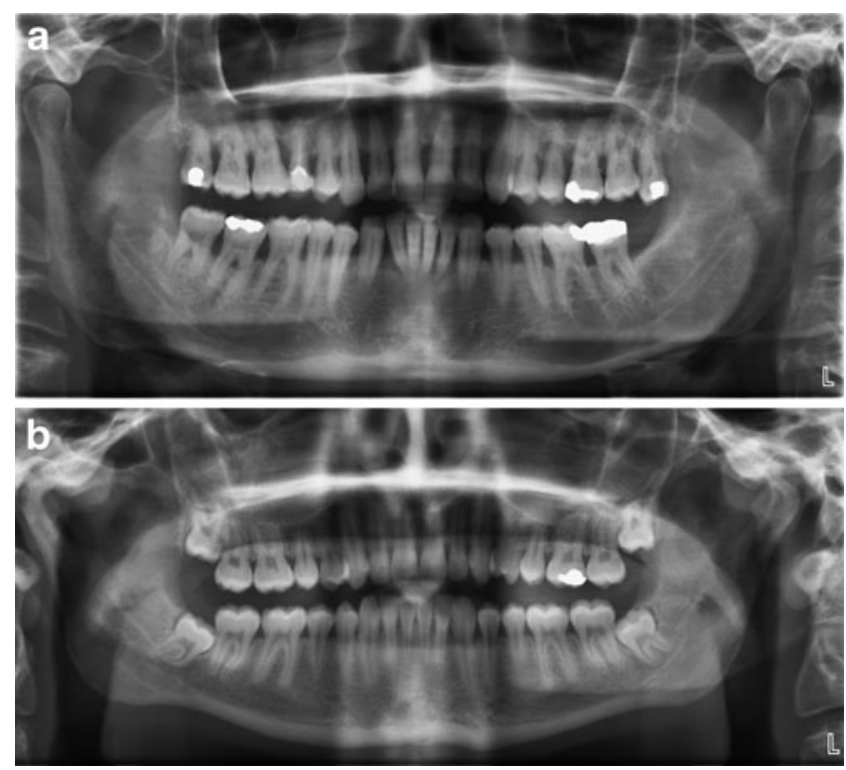

Fig. 3 Periodontitis (radiographic appearance). a A 42-year-old man with type 2 diabetes and generalised severe periodontitis. There is extensive alveolar bone loss (generally $50-75 \%$ of the root length) affecting the entire dentition, with an irregular (uneven) pattern of bone loss. Some of the teeth have lost nearly all their supporting alveolar bone as a result of periodontitis progression, e.g. the upper molars (both right and left), and the four lower incisors, all of which are grossly mobile and which are retained in the oral cavity only by the soft tissue attachment (having lost $100 \%$ of their bone support). b A 21-year-old man with no periodontitis. Alveolar bone levels are normal, with the crest of the alveolar bone being in close proximity to the cemento-enamel junction (the boundary between the enamel crown and the root). Contrast with appearance in Fig. 3a

alveolar bone loss compared with non-obese rats [19]. Adiposity can be regarded as a systemic disease that predisposes individuals to a variety of comorbidities and complications, and a number of studies have reported associations between obesity and periodontitis [20]. Analysis of NHANES III data identified that individuals with a BMI of $\geq 30 \mathrm{~kg} / \mathrm{m}^{2}$ had a significantly increased risk of periodontitis compared with individuals with a BMI of $18.5-24.9 \mathrm{~kg} / \mathrm{m}^{2}$ [21]. This relationship was potentially mediated by insulin resistance since, among those with a BMI of $\geq 27 \mathrm{~kg} / \mathrm{m}^{2}$, those who were in the highest quartile for insulin resistance had a significantly increased risk of severe periodontitis compared with those in the lowest quartile [22]. A recent meta-analysis revealed a significant association between periodontitis and obesity (OR 1.35; $95 \%$ CI $1.23,1.47$ ), and concluded that a higher prevalence of periodontitis should be expected among obese adults [23].

These findings raise the question of whether increased physical activity can reduce periodontitis risk. In the US Health Professionals Follow-up Study of 39,467 health professionals, an inverse linear relationship was identified between sustained physical activity and periodontitis, 
independent of known risk factors [24]. Another analysis of NHANES III data revealed that adults with higher levels of physical activity had significantly lower risk of periodontitis, with associations being strongest in non-smokers (but no association in smokers, suggesting that the harmful effects of smoking outweighed any benefit of physical activity) [25]. These various studies, while interesting, are limited in that they are generally cross-sectional/observation$\mathrm{al}$, and the temporal sequence of events is not clear (i.e. whether obesity precedes periodontitis). Prospective cohort studies are required to evaluate this area further.

\section{Impact of periodontitis on diabetes}

There has recently been much emphasis on the 'two-way' relationship between diabetes and periodontitis [26]. That is, not only is diabetes a risk factor for periodontitis, but periodontitis could have a negative effect on glycaemic control. The first clear evidence to support this hypothesis came from investigations of individuals in the Gila River Indian community. Severe periodontitis at baseline was associated with an increased risk of poor glycaemic control $\left(\mathrm{HbA}_{1 \mathrm{c}}>9.0 \%\right)$ at follow-up (minimum 2 years), suggesting that severe periodontitis was a risk factor for compromised diabetes management [27]. In addition, various studies have reported that the prevalence and severity of non-oral diabetes-related complications, including retinopathy, diabetic neuropathy, proteinuria and cardiovascular complications, are correlated with the severity of periodontitis [28-31].

Further studies of the Gila River Indian Community investigated the effect of periodontitis on the development of overt nephropathy, defined as macroalbuminuria and endstage renal disease (ESRD), in type 2 diabetes mellitus [32]. Periodontal status was assessed in 529 individuals aged $\geq 25$ years old who had type 2 diabetes mellitus, a glomerular filtration rate of $\geq 60 \mathrm{ml} \mathrm{min}{ }^{-1} 1.73 \mathrm{~m}^{-2}$, and no macroalbuminuria (urinary albumin:creatinine ratio $\geq 300 \mathrm{mg} / \mathrm{g}$ ). A total of 193 individuals developed macroalbuminuria (over a median follow-up of 9.4 years) and 68 developed ESRD (over a median follow-up of 14.9 years). After adjusting for age, sex, diabetes duration, BMI and smoking, the incidences of macroalbuminuria were 2.0, 2.1 and 2.6 times as high in those with moderate periodontitis, severe periodontitis, or who were edentulous, respectively, compared with those with no/mild periodontitis $(p<0.05)$. The incidences of ESRD were 2.3, 3.5 and 4.9 times as high for those with moderate or severe periodontitis or who were edentulous, respectively $(p<0.05)$. Thus, moderate and severe periodontitis and edentulousness predicted the development of overt nephropathy and ESRD in a 'dose-dependent' manner in individuals with type 2 diabetes mellitus and little or no pre-existing kidney disease [32].
The same researchers also investigated the effect of periodontitis on deaths from cardiovascular disease and diabetic nephropathy. In a prospective longitudinal study of 628 Pima Indians aged $\geq 35$ years with type 2 diabetes mellitus (median follow-up 11 years, during which 204 participants died), age- and sex-adjusted death rates (deaths per 1,000 person-years) were 3.7 for those with no or mild periodontitis, 19.6 for moderate periodontitis and 28.4 for severe periodontitis. Periodontitis was a predictor of deaths from ischaemic heart disease $(p=0.04)$ and diabetic nephropathy $(p<0.01)$. After adjusting for age, sex, diabetes duration, $\mathrm{HbA}_{1 \mathrm{c}}$, macroalbuminuria, BMI, cholesterol, hypertension, electrocardiogram abnormalities and smoking, diabetic individuals with severe periodontitis had 3.2 times the risk $(95 \%$ CI 1.1, 9.3) of cardiorenal mortality (ischaemic heart disease and diabetic nephropathy combined) compared with the reference group (no/mild periodontitis and moderate periodontitis combined) [33].

Acknowledging that the above studies were conducted in a specific, defined population, more recent research has investigated the impact of oral disease on subsequent cardiovascular disease in a general population of people with type 2 diabetes mellitus [34]. A total of 10,958 participants responded to two questions about the presence of oral disease (the number of natural teeth in their mouth, and the approximate number of days their gums had bled in the previous year) [35]; a lower number of remaining natural teeth and higher number of bleeding days were taken as indicators of poorer oral health. Despite the relatively crude oral health measures that were ascertained, age-, sex- and treatment allocation-adjusted analyses revealed that the group with no teeth were at almost twice the risk of death from all causes relative to those with $\geq 22$ teeth; people with 1-21 teeth had an intermediate risk. The authors of the paper postulated that inflammation resulting from poor oral health may have been implicated in the development of cardiovascular disease.

An intriguing area of recent investigation has focused on whether periodontitis plays a role in the incidence of diabetes. In a 7 year prospective study of 5,848 nondiabetic individuals aged 30-59 years, the effect of periodontitis on diabetes incidence (defined as fasting plasma glucose $>6.9 \mathrm{mmol} / \mathrm{l}$, equivalent to $>125 \mathrm{mg} / \mathrm{dl}$ ) was assessed [36]. In unadjusted analyses, moderate (pockets $3.5-5.5 \mathrm{~mm}$ ) and severe (pockets $>5.5 \mathrm{~mm}$ ) periodontitis were significantly associated with an increased risk of diabetes incidence, but significance was lost after adjusting for sex, smoking, BMI, triacylglycerol, hypertension, HDLcholesterol, and $\gamma$-glutamyl transpeptidase.

The impact of periodontitis on changes in $\mathrm{HbA}_{1 \mathrm{c}}$ was assessed in a prospective 5 year study of 2,973 non-diabetic individuals [37]. Those participants with the most advanced 
periodontitis at baseline demonstrated an approximately fivefold greater absolute increase in $\mathrm{HbA}_{1 \mathrm{c}}$ over the 5 years of the study compared with those with no periodontitis at baseline (change in $\mathrm{HbA}_{1 \mathrm{c}} 0.106 \pm 0.03 \%$ vs $0.023 \pm 0.02 \%$ ). This was the first study to report that periodontitis predicts the progression of $\mathrm{HbA}_{1 \mathrm{c}}$ among diabetes-free individuals and it is continuing to identify whether these subclinical changes in $\mathrm{HbA}_{1 \mathrm{c}}$ may translate into an increased risk of incident diabetes at 10 years.

Studies show that the incidences of macroalbuminuria and end-stage renal disease are increased twofold and threefold, respectively, in diabetic individuals who also have severe periodontitis. Furthermore, people with diabetes and severe periodontitis have a three times higher risk of cardiorenal mortality compared with those without severe periodontitis.

\section{What are the pathogenic mechanisms linking diabetes and periodontitis?}

Periodontitis is a complex chronic inflammatory disease in which inflammation in the periodontal tissues is stimulated by the long-term presence of the subgingival biofilm (dental plaque). The inflammatory response is characterised by dysregulated secretion of host-derived mediators of inflammation and tissue breakdown. The most extensively studied include IL-1 $\beta$, IL-6, prostaglandin $\mathrm{E}_{2}\left(\mathrm{PGE}_{2}\right)$, TNF- $\alpha$, receptor activator of nuclear factor $\kappa \mathrm{B}$ ligand (RANKL), and the matrix metalloproteinases (MMPs; particularly MMP-8, MMP-9 and MMP-13), as well as T cell regulatory cytokines (e.g. IL-12, IL-18) and the chemokines [38]. The complexity of cytokine networks in periodontal pathogenesis is becoming increasingly apparent, and it is clear that there is considerable heterogeneity in the nature of the inflammatory response between individuals. This heterogeneity exists not only between individuals, but also within individuals over time, and is influenced by genetic, epigenetic and environmental factors. The sum total of the inflammatory response in the periodontal tissues determines the pattern and rate of disease progression [39].

Inflammation is a central feature of the pathogenesis of diabetes and periodontitis

Both type 1 and type 2 diabetes mellitus are associated with elevated levels of systemic markers of inflammation [40]. The elevated inflammatory state in diabetes contributes to both microvascular and macrovascular complications, and it is clear that hyperglycaemia can result in the activation of pathways that increase inflammation, oxidative stress and apoptosis [41]. Elevated serum levels of IL- 6 and TNF- $\alpha$ have been demonstrated in diabetes and obesity [40], and serum levels of IL-6 and C-reactive protein (CRP) have been shown to predict future occurrence of type 2 diabetes mellitus [42]. Elevated levels of CRP are also associated with insulin resistance, type 2 diabetes mellitus and cardiovascular disease [43]. TNF- $\alpha$ and IL- 6 are the main inducers of acute-phase proteins, including CRP, and both have been shown to impair intracellular insulin signalling, potentially contributing to insulin resistance [44, 45]. Serum levels of IL-6 and CRP are also raised in patients with periodontitis, with IL-6 levels correlating with the extent of disease [46, 47]. The systemic inflammation that is associated with periodontal disease may therefore enhance the diabetic state. Adipokines may also contribute to susceptibility to both periodontitis and diabetes, and the proinflammatory properties of leptin may be particularly important in upregulating periodontal inflammation in people who are obese and/or have type 2 diabetes mellitus [48].

Diabetes increases inflammation in the periodontal tissues. For example, gingival crevicular fluid (GCF; a fluid exudate that flows from the gingival margins) levels of $\mathrm{PGE}_{2}$ and IL-1 $\beta$ are higher in type 1 diabetic patients with either gingivitis or periodontitis compared with those in non-diabetic individuals with the same level of periodontal disease [49]. In a study of type 2 diabetic patients, those with $\mathrm{HbA}_{1 \mathrm{c}}>8 \%$ had a significantly higher GCF IL- $1 \beta$ level compared with patients with $\mathrm{HbA}_{1 \mathrm{c}}<8 \%$, and both $\mathrm{HbA}_{1 \mathrm{c}}$ and random glucose were independent predictors of an elevated GCF IL-1 $\beta$ level [50].

When challenged with lipopolysaccharide, monocytes from type 1 diabetic patients produce significantly greater concentrations of TNF- $\alpha$, IL- $1 \beta$ and PGE $_{2}$ than monocytes from non-diabetic individuals $[49,51]$. Furthermore, studies have consistently demonstrated defects in polymorphonuclear leucocyte (PMN) activity in patients with diabetes, including impaired chemotaxis, phagocytosis and microbicidal functions [52]. PMNs require energy to function and these defects may be related to the metabolic changes that occur in diabetes [53]. Diabetic patients with severe periodontitis have been shown to have depressed PMN chemotaxis compared with diabetic individuals with mild periodontitis [54], as well as defective PMN apoptosis [55], which may lead to increased retention of PMNs in the periodontal tissue, leading to more tissue destruction by continued release of MMPs and reactive oxygen species (ROS). Diabetes prolongs the inflammatory response to Porphyromonas gingivalis (a periodontal pathogen commonly found in the biofilm of patients with advanced periodontitis), with increased production of TNF- $\alpha$ [56]. Periodontal treatment has been shown to reduce serum levels 
of inflammatory mediators, including IL-6, TNF- $\alpha$, CRP and MMPs, in patients with and without diabetes [47, 57-60]

Accumulation of AGEs in the periodontal tissues is also likely to play a role in upregulating periodontal inflammation in individuals with diabetes. Binding of AGE to its receptor (RAGE) results in the upregulated production of inflammatory mediators such as IL-1 $\beta$, TNF- $\alpha$ and IL-6 [61]. AGE formation results in the production of ROS and enhances oxidant stress, and the subsequent endothelial cell changes that occur contribute to the vascular injury implicated in many diabetes complications [62]. AGEs also enhance the respiratory burst in PMNs [63], which has the potential to significantly increase local tissue damage in periodontitis. Furthermore, AGEs have detrimental effects on bone metabolism, leading to impaired repair and bone formation [64] and decreased extracellular matrix production [65]. Apoptosis may also play a role in the increased susceptibility to periodontitis associated with diabetes, and apoptosis of matrix-producing cells may limit the opportunities for repair in inflamed tissues. Induction of tissue injury by inoculation with $P$. gingivalis resulted in significantly higher fibroblast apoptosis in diabetic mice compared with non-diabetic mice [66], indicating another mechanism by which diabetes can interfere with the capacity for repair in inflamed periodontal tissues. The various inflammatory pathways postulated to link diabetes and periodontitis are illustrated in Fig. 4.

\section{Is there a relationship between the oral microbiota and diabetes?}

Compared with the large number of studies that have investigated the role of inflammatory mechanisms in the link between periodontitis and diabetes, relatively few have investigated relationships between the oral microbiota and diabetes. In one study, recovery of several periodontal pathogens, including Aggregatibacter actinomycetemcomitans, Campylobacter rectus, Capnocytophaga spp, Eikenella corrodens, Fusobacterium nucleatum and Prevotella intermedia, was similar in both diabetic and non-diabetic participants, but significantly more individuals with diabetes harboured P. gingivalis [67]. Similarly, in a study of young Japanese individuals with type 1 diabetes mellitus, a greater proportion of participants with periodontitis harboured $P$. gingivalis and $P$. intermedia than those who were periodontally healthy [68]. These studies indicate that there are probably subtle differences in the microbial composition of the subgingival biofilm between individuals with diabetes and those without, but the clinical relevance of this is not clear. Such differences may arise from the effect of diabetes in altering the local environment within the periodontal pocket such that the growth of certain bacterial species is favoured.

The contribution of oral bacteria to adiposity has also been assessed. In a study of 313 women with BMI of $27-$ $32 \mathrm{~kg} / \mathrm{m}^{2}$ (compared with 232 healthy individuals), 98.4\%

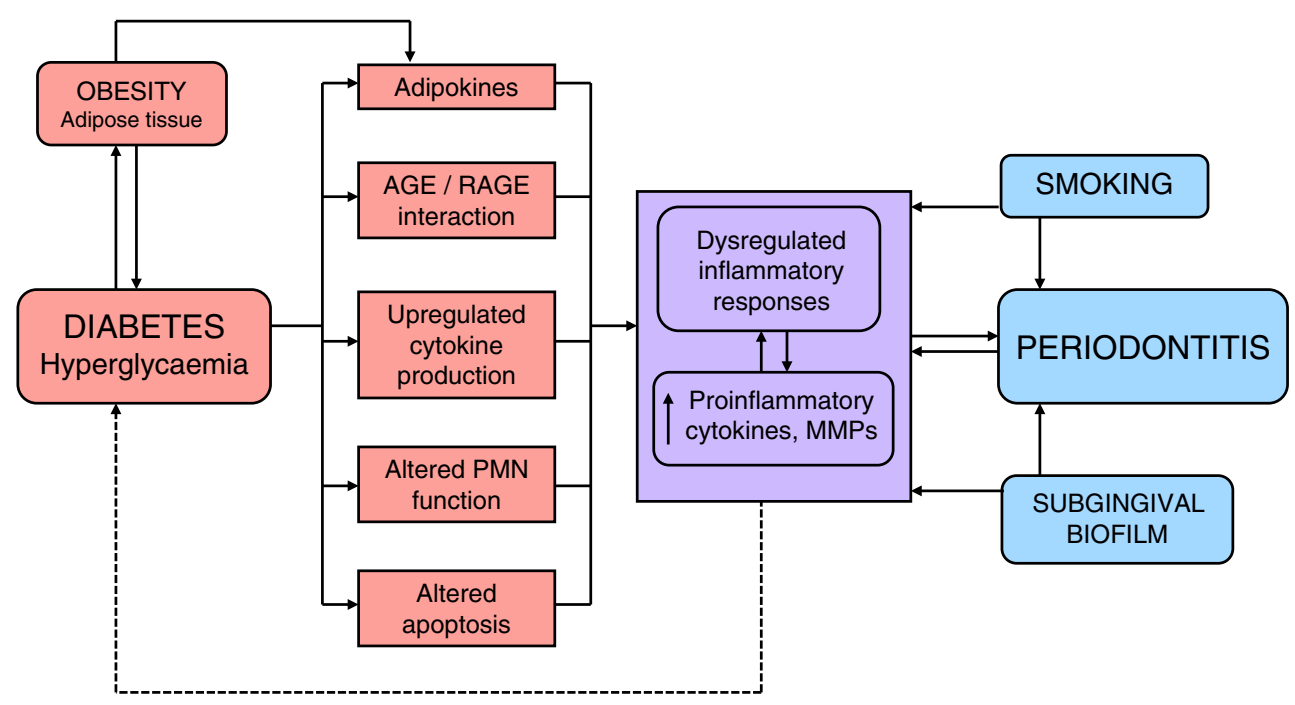

Fig. 4 Schematic representation of the proposed two-way relationship between diabetes and periodontitis. Exacerbated and dysregulated inflammatory responses are at the heart of the proposed two-way interaction between diabetes and periodontitis (purple box), and the hyperglycaemic state results in various proinflammatory effects that impact on multiple body systems, including the periodontal tissues. Adipokines produced by adipose tissue include proinflammatory mediators such as TNF- $\alpha$, IL- 6 and leptin. The hyperglycaemic state results in deposition of AGEs in the periodontal tissues (as well as elsewhere in the body), and binding of the receptor for AGE (RAGE) results in local cytokine release and altered inflammatory responses. Neutrophil function is also altered in the diabetic state, resulting in enhancement of the respiratory burst and delayed apoptosis (leading to increased periodontal tissue destruction). Local production of cytokines in the periodontal tissues may, in turn, affect glycaemic control through systemic exposure and an impact on insulin signalling (dotted arrow). All of these factors combine to contribute to dysregulated inflammatory responses that develop in the periodontal tissues in response to the chronic challenge by bacteria in the subgingival biofilm, and which are further exacerbated by smoking 
of the overweight women could be identified by the presence of the periodontal pathogen Selenomonas noxia at levels $>1.05 \%$ of the total bacterial population [69]. $S$. noxia $>1.05 \%$ had a sensitivity of $98 \%$ and specificity of $80 \%$ of predicting obesity, leading to the interesting question of whether oral bacteria are involved in the pathology that leads to obesity. The tenets of 'infectobesity' suggest that the gut microbiota of obese individuals may be more efficient at extracting energy from a given diet than that of lean individuals [70, 71], and gut microorganisms can affect host metabolism, influencing inflammation and insulin resistance [72]. The role of swallowed periodontal bacteria in contributing to these effects has yet to be established, but it is clear from animal studies that influencing the intestinal microbiota (for example, through the use of prebiotics, specific nutrients, or natural antibiotics) could potentially change satiety and insulin resistance and be of benefit in the management of diabetes $[73,74]$.

\section{Periodontal treatment is associated with improved glycaemic control}

Several meta-analyses have confirmed that effective periodontal therapy can result in reduced $\mathrm{HbA}_{1 \mathrm{c}}$. The first reported on ten interventional studies with a combined population of 456 patients; the authors identified a weighted mean reduction in $\mathrm{HbA}_{1 \mathrm{c}}$ of $0.66 \%$ as a result of periodontal therapy (though this failed to achieve statistical significance) [75]. In 2008, a meta-analysis of nine studies involving 485 patients reported a significant reduction of $\mathrm{HbA}_{1 \mathrm{c}}$ of $0.46 \%$ following periodontal treatment [76]. In 2010, a meta-analysis of five studies involving 371 patients also reported a significant weighted mean reduction in $\mathrm{HbA}_{1 \mathrm{c}}$ of $0.40 \%$ over a follow-up period of 3-9 months after periodontal therapy [77]. The authors of these meta-analyses all commented on the heterogeneity of the data, different methodologies having being used in the different studies. Most recently, the Cochrane Collaboration has reported on studies that investigated the relationship between periodontal treatment and glycaemic control in people with diabetes [78]. Three studies were included in this meta-analysis which reported a significant reduction in $\mathrm{HbA}_{1 \mathrm{c}}$ of $0.40 \%$ 3-4 months after conventional periodontal therapy (Fig. 5). The findings of these metaanalyses are supported a recent population-based study of over 5,000 individuals with diabetes [79] reporting that patients who received at least one episode of periodontal surgery (an intense form of periodontal treatment, not routinely undertaken in all patients with periodontitis) had $\mathrm{HbA}_{1 \mathrm{c}}$ levels that were $0.25 \%$ lower than patients who did not undergo periodontal surgery [80-82].
Taken collectively, the evidence supports the notion that improvements in metabolic control can be anticipated following effective treatment of periodontitis (although there are few studies available, and some studies lack power). The mechanisms by which this occurs are not yet clear, but probably relate to reduced systemic inflammation (e.g. reduced serum levels of mediators such as TNF- $\alpha$ and IL-6) following the treatment and resolution of periodontal inflammation. Larger randomised trials are warranted to investigate this further. These observations are important because reductions in $\mathrm{HbA}_{1 \mathrm{c}}$ are associated with a reduced risk of diabetes complications. For example, each $1 \%$ reduction in $\mathrm{HbA}_{1 \mathrm{c}}$ has been associated with reductions in risk of $21 \%$ for any endpoint related to diabetes, $21 \%$ for deaths related to diabetes, $14 \%$ for myocardial infarction and $37 \%$ for microvascular complications [83].

Meta-analyses indicate that $\mathrm{HbA}_{1 \mathrm{c}}$ reductions of around $0.4 \%$ can be anticipated following effective periodontal therapy.

\section{The healthcare implications of the link between diabetes and periodontitis}

In 2000, the US Surgeon General referred to a 'silent epidemic' of oral and dental diseases, and stressed the importance of oral health as being essential for general health and well-being [84]. In 2007, the WHO Executive Board acknowledged the intrinsic link between oral health, general health and quality of life [85]. A recent editorial in The Lancet stated that 'oral health is a neglected area of global health' and indicated that promoting and improving oral health should be part of the routine business of healthcare policymakers and clinicians [86]. Because poor oral health primarily affects morbidity rather than mortality, policymakers and governments have unfortunately tended to view oral diseases as less important than more lifethreatening diseases. However, oral diseases are highly prevalent and are associated with significant morbidity: dental caries is one of the most prevalent diseases worldwide, severe periodontitis typically affects up to $15 \%$ of most adult populations, oral cancer is the eighth most common cancer worldwide, and approximately half of people who are HIV positive have oral fungal, bacterial or viral infections [86].

The management of diabetes is complex and the prevention of cardiovascular and microvascular disease, through early detection and management of complications, are key components. Lifestyle intervention, education, selfmanagement and self-monitoring are particularly important, 


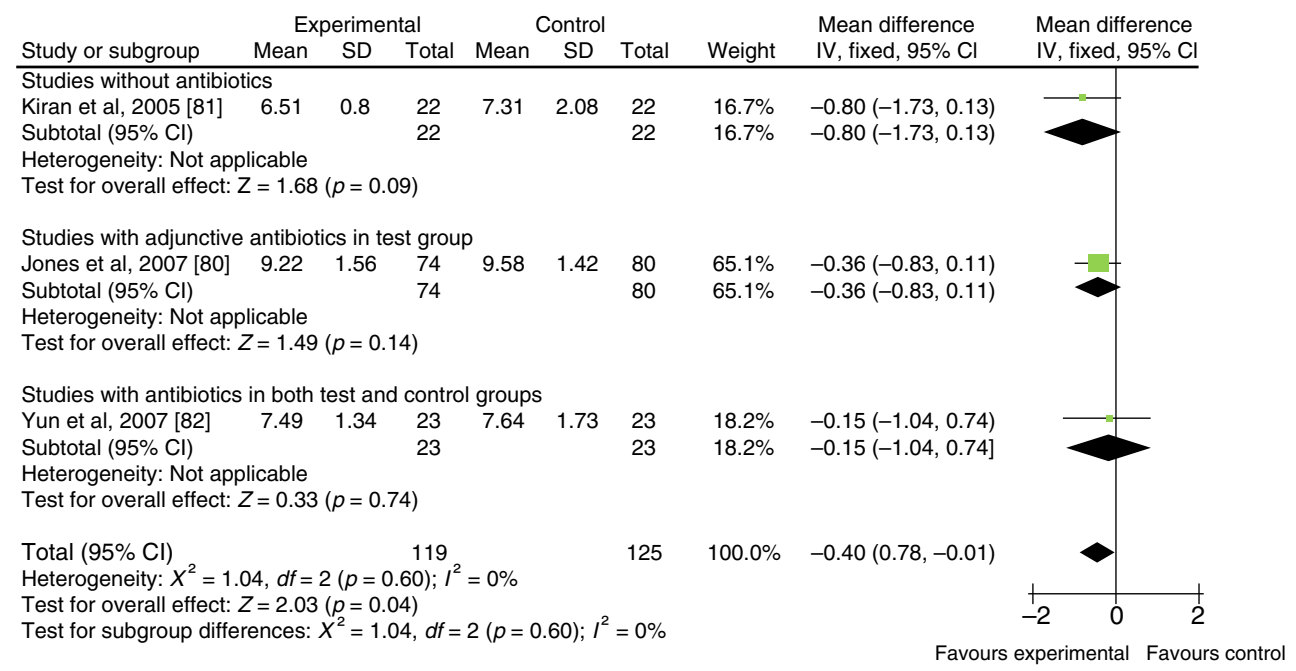

Fig. 5 Forest plot to indicate outcome of periodontitis treatment on $\mathrm{HbA}_{1 \mathrm{c}}$ levels after 3-4 months. The effect for the mean percentage difference as a result of periodontal treatment was $-0.40 \%(95 \%$ CI $0.78 \%,-0.01 \%), n=244$, representing a statistically significant reduction in $\mathrm{HbA}_{1 \mathrm{c}}(p=0.04)$ as a result of the periodontal treatment. IV, inverse

in addition to treatments to reduce blood glucose, blood pressure and lipids [87]. Similar to diabetes, current treatment philosophies for periodontitis strongly emphasise self-management through patient education. A supportive and facilitative approach by the dental team is essential, but there must be a clear understanding that patient-performed plaque control is the vehicle by which to control the inflammation which drives periodontal tissue destruction. Structured education programmes are effective in the management of diabetes [88-90], and similar programmes are being developed for the management of periodontitis $[91,92]$. These education programmes all emphasise the importance of engaging with the patient and ensuring that patients develop self-efficacy in managing their disease as the means to effect the lifelong behavioural changes that are required for the successful management of both conditions. The importance of self-efficacy in the control of diabetes and oral hygiene has been demonstrated in a population of diabetic individuals in Finland. Those individuals with better tooth-brushing self-efficacy had lower plaque scores (as might be expected) and lower $\mathrm{HbA}_{1 \mathrm{c}}$ levels compared with those who had poorer self-efficacy [93]. Furthermore, diabetic participants who managed their gingivitis successfully also tended to report better glycaemic control and had lower mean $\mathrm{HbA}_{1 \mathrm{c}}$ levels $(8.1 \pm 1.5 \%)$ compared with participants who did not manage their gingivitis effectively $(9.0 \pm 1.9 \%)$ [94]. These studies suggest that there are common determinants for both dental and diabetes selfcare that could be exploited for improved management of both conditions. variance (from Simpson TC, Needleman I, Wild SH, Moles DR, Mills EJ Treatment of periodontal disease for glycaemic control in people with diabetes. Cochrane Database Syst Rev, 2010, Issue 5. Copyright Cochrane Collaboration, reproduced with permission)

There is, therefore, a cogent argument for involving the dental team in the management of diabetes. Indeed, the dental team is well placed to screen patients for diabetes by virtue of the fact that many people visit their dentist regularly (e.g. every 6 months, often more frequently than they visit their medical practitioner), and the intra-oral findings may raise suspicion of undiagnosed diabetes. The dental team (particularly dental hygienists) are very adept and experienced in instituting behavioural changes in their patients, and may represent an untapped source of support for medical colleagues in this role.

The parallels between patient responsibilities in managing their diabetes or periodontitis are striking. Successful treatment of periodontitis involves structured patient education and empowerment, establishing treatment needs and setting goals, personalised biofeedback (e.g. reductions in gingival bleeding and probing depths), frequent recall, and the involvement of various members of the dental team, particularly dental hygienists.

\section{Conclusions}

Epidemiological studies confirm that diabetes is a significant risk factor for periodontitis, and the risk of periodontitis is greater if glycaemic control is poor; people with poorly controlled diabetes (who are also 
most at risk for the other macrovascular and microvascular complications) are at an increased risk of periodontitis and alveolar bone loss [5, 95]. Given the predicted increases in the prevalence of diabetes over the next few decades, we will probably see a reversal of the hitherto experienced reductions in the prevalence of periodontitis (associated with less smoking and better oral healthcare behaviours over recent years) as a result of large increases in the number of people with diabetes [96]. Controlling diabetes (i.e. improving glycaemic control) is likely to reduce the risk and severity of periodontitis. Furthermore, evidence suggests that resolution of periodontal inflammation can improve metabolic control (with reported $\mathrm{HbA}_{1 \mathrm{c}}$ reductions of approximately $0.4 \%$ ), though large, multi-centre, randomised controlled trials are needed to further validate these findings.

\section{Key points}

- People with poorly controlled diabetes (both type 1 and type 2 diabetes mellitus, both adults and children) must be considered at risk for periodontitis, and people with diabetes should be informed of this risk.

- Early diagnosis and prevention are of fundamental importance to avoid the largely irreversible tissue loss that occurs in periodontitis, and early referral of adults and children with poorly controlled diabetes to dental clinicians is indicated for periodontal screening.

- Periodontal therapy in patients with diabetes is associated with improvements in glycaemic control $\left(\mathrm{HbA}_{1 \mathrm{c}}\right.$ reductions of approximately $0.4 \%$ ) that may be clinically relevant in the management of diabetes.

- Oral health should be promoted in people with diabetes as an integral component of their overall diabetes management.

- Closer collaboration between medical and dental clinical teams is necessary for the joint management of people with diabetes and periodontitis, and contact with dentists is important after the diagnosis of diabetes.

Acknowledgements This manuscript developed as a consensus following the European Workshop in Periodontal Diseases and Diabetes that was held in London, on 26 May 2010, and was made possible by an unrestricted educational grant from Colgate Palmolive Europe. Seven experts (four representing the field of periodontology and three the field of diabetes) addressed the issues surrounding the links between periodontitis and diabetes. The aims of the Workshop were to consider the evidence for the links between diabetes and periodontitis, to address the health implications of these associations, and to provide guidance for clinicians. The authors would like to acknowledge the assistance of R. Pugh (freelance health writer) for recording minutes of the discussions at the Workshop.
Contribution statement PMP was the main contributor to the concept, design, literature analysis, workshop discussions, and drafting and revising manuscript. ALA, DH, SJ, AK, KM and RT contributed substantially to the concept, design, literature analysis, workshop discussions, and drafting and revising manuscript. All authors gave final approval of version to be published.

Duality of interest statement The authors declare that there is no duality of interest associated with this manuscript.

Open Access This article is distributed under the terms of the Creative Commons Attribution Noncommercial License which permits any noncommercial use, distribution, and reproduction in any medium, provided the original author(s) and source are credited.

\section{References}

1. Fox CH (1992) New considerations in the prevalence of periodontal disease. Curr Opin Dent 2:5-11

2. Fox CH, Jette AM, McGuire SM, Feldman HA, Douglass CW (1994) Periodontal disease among New England elders. J Periodontol 65:676-684

3. Kelly M, Steele J, Nuttall N et al (2000) The condition of supporting structures. In: Walker A, Cooper I (eds) Adult dental health survey: oral health in the United Kingdom 1998. The Stationery Office, London, pp 123-146

4. O'Dowd LK, Durham J, McCracken GI, Preshaw PM (2010) Patients' experiences of the impact of periodontal disease. J Clin Periodontol 37:334-339

5. Pihlstrom BL, Michalowicz BS, Johnson NW (2005) Periodontal diseases. Lancet 366:1809-1820

6. Grossi SG, Genco RJ, Machtei EE et al (1995) Assessment of risk for periodontal disease. II. Risk indicators for alveolar bone loss. J Periodontol 66:23-29

7. Salvi GE, Carollo-Bittel B, Lang NP (2008) Effects of diabetes mellitus on periodontal and peri-implant conditions: update on associations and risks. J Clin Periodontol 35:398409

8. Chavarry NGM, Vettore MV, Sansone C, Sheiham A (2009) The relationship between diabetes mellitus and destructive periodontal disease: a meta-analysis. Oral Health Prev Dent 7:107-127

9. Khader YS, Dauod AS, El-Qaderi SS, Alkafajei A, Batayha WQ (2006) Periodontal status of diabetics compared with nondiabetics: a meta-analysis. J Diabetes Complicat 20:59-68

10. Mealey BL, Ocampo GL (2007) Diabetes mellitus and periodontal disease. Periodontol 2000 44:127-153

11. Tsai C, Hayes C, Taylor GW (2002) Glycemic control of type 2 diabetes and severe periodontal disease in the US adult population. Community Dent Oral Epidemiol 30:182-192

12. Nelson RG, Shlossman M, Budding LM et al (1990) Periodontal disease and NIDDM in Pima Indians. Diabetes Care 13:836-840

13. Taylor GW, Burt BA, Becker MP, Genco RJ, Shlossman M (1998) Glycemic control and alveolar bone loss progression in type 2 diabetes. Ann Periodontol 3:30-39

14. Emrich LJ, Shlossman M, Genco RJ (1991) Periodontal disease in non-insulin dependent diabetes mellitus. J Periodontol 62:123-131

15. Cianciola LJ, Park PH, Bruck E, Mosovich L, Genco RJ (1982) Prevalence of periodontal disease in insulin-dependent mellitus (juvenile diabetes). J Am Dent Assoc 104:653-660

16. Lalla E, Cheng B, Lal S et al (2007) Diabetes mellitus promotes periodontal destruction in children. J Clin Periodontol 34:294-298 
17. Loe H (1993) Periodontal disease. The sixth complication of diabetes mellitus. Diabetes Care 16:329-334

18. American Diabetes Association Expert Committee on the Diagnosis and Classification of Diabetes Mellitus (2003) Report of the Expert Committee on the Diagnosis and Classification of Diabetes Mellitus. Diabetes Care 26:S5-S20

19. Perlstein MI, Bissada NF (1977) Influence of obesity and hypertension on the severity of periodontitis in rats. Oral Surg Oral Med Oral Pathol 43:707-719

20. Pischon N, Heng N, Bernimoulin JP, Kleber BM, Willich SN, Pischon T (2007) Obesity, inflammation, and periodontal disease. J Dent Res 86:400-409

21. Al-Zahrani MS, Bissada NF, Borawskit EA (2003) Obesity and periodontal disease in young, middle-aged, and older adults. J Periodontol 74:610-615

22. Genco RJ, Grossi SG, Ho A, Nishimura F, Murayama Y (2005) A proposed model linking inflammation to obesity, diabetes, and periodontal infections. J Periodontol 76:2075-2084

23. Chaffee BW, Weston SJ (2010) Association between chronic periodontal disease and obesity: a systematic review and metaanalysis. J Periodontol 81:1708-1724

24. Merchant AT, Pitiphat W, Rimm EB, Joshipura K (2003) Increased physical activity decreases periodontitis risk in men. Eur J Epidemiol 18:891-898

25. Al-Zahrani MS, Borawski EA, Bissada NF (2005) Increased physical activity reduces prevalence of periodontitis. J Dent 33:703-710

26. Taylor GW (2001) Bidirectional interrelationships between diabetes and periodontal diseases: an epidemiologic perspective. Ann Periodontol 6:99-112

27. Taylor GW, Burt BA, Becker MP et al (1996) Severe periodontitis and risk for poor glycemic control in patients with non-insulindependent diabetes mellitus. J Periodontol 67:1085-1093

28. Karjalainen KM, Knuuttila ML, von Dickhoff KJ (1994) Association of the severity of periodontal disease with organ complications in type 1 diabetic patients. J Periodontol 65:1067-1072

29. Moore PA, Weyant RJ, Mongelluzzo MB et al (1998) Type 1 diabetes mellitus and oral health: assessment of tooth loss and edentulism. J Public Health Dent 58:135-142

30. Moore PA, Weyant RJ, Mongelluzzo MB et al (1999) Type 1 diabetes mellitus and oral health: assessment of periodontal disease. J Periodontol 70:409-417

31. Thorstensson H, Kuylenstierna J, Hugoson A (1996) Medical status and complications in relation to periodontal disease experience in insulin-dependent diabetics. J Clin Periodontol 23:194-202

32. Shultis WA, Weil EJ, Looker HC et al (2007) Effect of periodontitis on overt nephropathy and end-stage renal disease in type 2 diabetes. Diabetes Care 30:306-311

33. Saremi A, Nelson RG, Tulloch-Reid M et al (2005) Periodontal disease and mortality in type 2 diabetes. Diabetes Care 28:27-32

34. The ADVANCE Collaborative Group (2001) Study rationale and design of ADVANCE: action in diabetes and vascular diseasepreterax and diamicron MR controlled evaluation. Diabetologia 44:1118-1120

35. Li Q, Chalmers J, Czernichow S et al (2010) Oral disease and subsequent cardiovascular disease in people with type 2 diabetes: a prospective study based on the Action in Diabetes and Vascular Disease: Preterax and Diamicron Modified-Release Controlled Evaluation (ADVANCE) trial. Diabetologia 53:2320-2327

36. Ide R, Hoshuyama T, Wilson D, Takahashi K, Higashi T (2011) Periodontal disease and incident diabetes: a seven-year study. J Dent Res 90:41-46

37. Demmer RT, Desvarieux M, Holtfreter B et al (2010) Periodontal status and A1C change: longitudinal results from the study of health in Pomerania (SHIP). Diabetes Care 33:1037-1043

38. Preshaw PM, Taylor JJ (2011) How has research into cytokine interactions and their role in driving immune responses impacted our understanding of periodontitis? J Clin Periodontol 38(suppl 11):60-84

39. Kinane DF, Preshaw PM, Loos BG (2011) Host-response: understanding the cellular and molecular mechanisms of host-microbial interactions - Consensus of the Seventh European Workshop on Periodontology. J Clin Periodontol 38(suppl 11):44-48

40. Dandona P, Aljada A, Bandyopadhyay A (2004) Inflammation: the link between insulin resistance, obesity and diabetes. Trends Immunol 25:4-7

41. Brownlee M (2005) The pathobiology of diabetic complications: a unifying mechanism. Diabetes 54:1615-1625

42. Schmidt MI, Duncan BB, Sharrett AR et al (1999) Markers of inflammation and prediction of diabetes mellitus in adults (Atherosclerosis Risk in Communities study): a cohort study. Lancet 353:1649-1652

43. Nesto R (2004) C-reactive protein, its role in inflammation, type 2 diabetes and cardiovascular disease, and the effects of insulinsensitizing treatment with thiazolidinediones. Diabetic Med 21:810-817

44. Hotamisligil GS (2000) Molecular mechanisms of insulin resistance and the role of the adipocyte. Int $\mathrm{J}$ Obes Relat Metab Disord 24(Suppl 4):S23-S27

45. Rotter V, Nagaev I, Smith U (2003) Interleukin-6 (IL-6) induces insulin resistance in 3T3-L1 adipocytes and is, like IL-8 and tumor necrosis factor- $\alpha$, overexpressed in human fat cells from insulin-resistant subjects. J Biol Chem 278:45777-45784

46. Loos BG (2005) Systemic markers of inflammation in periodontitis. J Periodontol 76:2106-2115

47. Paraskevas S, Huizinga JD, Loos BG (2008) A systematic review and meta-analyses on $\mathrm{C}$-reactive protein in relation to periodontitis. J Clin Periodontol 35:277-290

48. Preshaw PM, Foster N, Taylor JJ (2007) Cross-susceptibility between periodontal disease and type 2 diabetes mellitus: an immunobiological perspective. Periodontol 2000 45:138-157

49. Salvi GE, Yalda B, Collins JG et al (1997) Inflammatory mediator response as a potential risk marker for periodontal diseases in insulin-dependent diabetes mellitus populations. J Periodontol $68: 127-135$

50. Engebretson SP, Hey-Hadavi J, Ehrhardt FJ et al (2004) Gingival crevicular fluid levels of interleukin- $1 \beta$ and glycemic control in patients with chronic periodontitis and type 2 diabetes. J Periodontol 75:1203-1208

51. Salvi GE, Collins JG, Yalda B, Arnold RR, Lang NP, Offenbacher $\mathrm{S}$ (1997) Monocytic TNF- $\alpha$ secretion patterns in IDDM patients with periodontal diseases. J Clin Periodontol 24:8-16

52. Alba-Loureiro TC, Munhoz CD, Martins JO et al (2007) Neutrophil function and metabolism in individuals with diabetes mellitus. Braz J Med Biol Res 40:1037-1044

53. Alba-Loureiro TC, Hirabara SM, Mendonca JR, Curi R, PithonCuri TC (2006) Diabetes causes marked changes in function and metabolism of rat neutrophils. J Endocrinol 188:295-303

54. Manouchehr-Pour M, Spagnuolo PJ, Rodman HM, Bissada NF (1981) Impaired neutrophil chemotaxis in diabetic patients with severe periodontitis. J Dent Res 60:729-730

55. Graves DT, Liu R, Alikhani M, Al-Mashat H, Trackman PC (2006) Diabetes-enhanced inflammation and apoptosis - impact on periodontal pathology. J Dent Res 85:15-21

56. Naguib G, Al-Mashat H, Desta T, Graves DT (2004) Diabetes prolongs the inflammatory response to a bacterial stimulus through cytokine dysregulation. J Invest Dermatol 123:87-92

57. D'Aiuto F, Parkar M, Andreou G et al (2004) Periodontitis and systemic inflammation: control of the local infection is associated with a reduction in serum inflammatory markers. J Dent Res $83: 156-160$

58. Iwamoto Y, Nishimura F, Nakagawa M et al (2001) The effect of antimicrobial periodontal treatment on circulating tumor necrosis 
factor-alpha and glycated hemoglobin level in patients with type 2 diabetes. J Periodontol 72:774-778

59. Marcaccini AM, Meschiari CA, Sorgi CA et al (2009) Circulating interleukin- 6 and high-sensitivity C-reactive protein decrease after periodontal therapy in otherwise healthy subjects. J Periodontol 80:594-602

60. O'Connell PAA, Taba M, Nomizo A et al (2008) Effects of periodontal therapy on glycemic control and inflammatory markers. J Periodontol 79:774-783

61. Lalla E, Lamster IB, Stern DM, Schmidt AM (2001) Receptor for advanced glycation end products, inflammation, and accelerated periodontal disease in diabetes: mechanisms and insights into therapeutic modalities. Ann Periodontol 6:113-118

62. Vlassara H (2001) The AGE-receptor in the pathogenesis of diabetic complications. Diabetes Metab Res 17:436-443

63. Wong RK, Pettit AI, Quinn PA, Jennings SC, Davies JE, Ng LL (2003) Advanced glycation end products stimulate an enhanced neutrophil respiratory burst mediated through the activation of cytosolic phospholipase A2 and generation of arachidonic acid. Circulation 108:1858-1864

64. Santana RB, Xu L, Chase HB, Amar S, Graves DT, Trackman PC (2003) A role for advanced glycation end products in diminished bone healing in type 1 diabetes. Diabetes 52:1502-1510

65. Cortizo AM, Lettieri MG, Barrio DA, Mercer N, Etcheverry SB, McCarthy AD (2003) Advanced glycation end-products (AGEs) induce concerted changes in the osteoblastic expression of their receptor RAGE and in the activation of extracellular signalregulated kinases (ERK). Mol Cell Biochem 250:1-10

66. Liu R, Desta T, He H, Graves DT (2004) Diabetes alters the response to bacteria by enhancing fibroblast apoptosis. Endocrinology 145:2997-3003

67. Thorstensson H, Dahlen G, Hugoson A (1995) Some suspected periodontopathogens and serum antibody response in adult longduration insulin-dependent diabetics. J Clin Periodontol 22:449458

68. Takahashi K, Nishimura F, Kurihara M et al (2001) Subgingival microflora and antibody responses against periodontal bacteria of young Japanese patients with type 1 diabetes mellitus. J Int Acad Periodontol 3:104-111

69. Goodson JM, Groppo D, Halem S, Carpino E (2009) Is obesity an oral bacterial disease? J Dent Res 88:519-523

70. Ley RE, Turnbaugh PJ, Klein S, Gordon JI (2006) Microbial ecology: human gut microbes associated with obesity. Nature 444:1022-1023

71. Turnbaugh PJ, Ley RE, Mahowald MA, Magrini V, Mardis ER, Gordon JI (2006) An obesity-associated gut microbiome with increased capacity for energy harvest. Nature 444:1027-1031

72. Ley RE (2010) Obesity and the human microbiome. Curr Opin Gastroenterol 26:5-11

73. Gubern C, Lopez-Bermejo A, Biarnes J, Vendrell J, Ricart W, Fernandez-Real JM (2006) Natural antibiotics and insulin sensitivity: the role of bactericidal/permeability-increasing protein. Diabetes 55:216-224

74. Vrieze A, Holleman F, Zoetendal EG, de Vos WM, Hoekstra JBL, Nieuwdorp M (2010) The environment within: how gut microbiota may influence metabolism and body composition. Diabetologia 53:606-613

75. Janket SJ, Wightman A, Baird AE, Van Dyke TE, Jones JA (2005) Does periodontal treatment improve glycemic control in diabetic patients? A meta-analysis of intervention studies. J Dent Res 84:1154-1159

76. Darre L, Vergnes JN, Gourdy P, Sixou M (2008) Efficacy of periodontal treatment on glycaemic control in diabetic patients: a meta-analysis of interventional studies. Diabetes Metab 34:497-506
77. Teeuw WJ, Gerdes VEA, Loos BG (2010) Effect of periodontal treatment on glycemic control of diabetic patients: a systematic review and meta-analysis. Diabetes Care 33:421-427

78. Simpson TC, Needleman I, Wild SH, Moles DR, Mills EJ (2010) Treatment of periodontal disease for glycaemic control in people with diabetes. Cochrane Database Syst Rev. doi:10.1002/ 14651858.CD004714.pub2

79. Spangler L, Reid RJ, Inge R et al (2010) Cross-sectional study of periodontal care and glycosylated hemoglobin in an insured population. Diabetes Care 33:1753-1758

80. Jones JA, Miller DR, Wehler CJ et al (2007) Does periodontal care improve glycemic control? The Department of Veterans Affairs Dental Diabetes Study. J Clin Periodontol 34:46-52

81. Kiran M, Arpak N, Unsal E, Erdogan MF (2005) The effect of improved periodontal health on metabolic control in type 2 diabetes mellitus. J Clin Periodontol 32:266-272

82. Yun F, Firkova EI, Jun-Qi L, Xun H (2007) Effect of non-surgical periodontal therapy on patients with type 2 diabetes mellitus. Folia Medica (Plovdiv) 49:32-36

83. Stratton IM, Adler AI, Neil HA et al (2000) Association of glycaemia with macrovascular and microvascular complications of type 2 diabetes (UKPDS 35): prospective observational study. BMJ 321:405-412

84. US Department of Health and Human Services (2000) Oral health in America: a report of the surgeon general. US Department of Health and Human Services, National Institute of Dental and Craniofacial Research, National Institutes of Health, Rockville

85. WHO (2007) Oral health: action plan for promotion and integrated disease prevention (EB120/10). 120th Session, 22-30 January. WHO, Geneva

86. Lancet (2009) Oral health: prevention is key. Lancet 373:1

87. Home P, Mant J, Diaz J, Turner C (2008) Guideline Development Group. Management of type 2 diabetes: summary of updated NICE guidance. BMJ 336:1306-1308

88. Davies MJ, Heller S, Khunti K, Skinner TC (2008) The DESMOND educational intervention. Chronic Illn 4:38-40

89. Deakin TA, Cade JE, Williams R, Greenwood DC (2006) Structured patient education: the diabetes X-PERT Programme makes a difference. Diabetic Med 23:944-954

90. Gillett M, Dallosso HM, Dixon S et al (2010) Delivering the diabetes education and self management for ongoing and newly diagnosed (DESMOND) programme for people with newly diagnosed type 2 diabetes: cost effectiveness analysis. BMJ 341:c4093

91. Jonsson B, Ohrn K, Oscarson N, Lindberg P (2009) The effectiveness of an individually tailored oral health educational programme on oral hygiene behaviour in patients with periodontal disease: a blinded randomized-controlled clinical trial (one-year follow-up). J Clin Periodontol 36:1025-1034

92. Jonsson B, Ohrn K, Oscarson N, Lindberg P (2009) An individually tailored treatment programme for improved oral hygiene: introduction of a new course of action in health education for patients with periodontitis. Int J Dent Hyg 7:166-175

93. Syrjala AM, Kneckt MC, Knuuttila ML (1999) Dental selfefficacy as a determinant to oral health behaviour, oral hygiene and $\mathrm{HbA} 1 \mathrm{c}$ level among diabetic patients. J Clin Periodontol 26:616-621

94. Kneckt MC, Syrjala AM, Knuuttila ML (2000) Attributions to dental and diabetes health outcomes. J Clin Periodontol 27:205-211

95. Soskolne WA, Klinger A (2001) The relationship between periodontal diseases and diabetes: an overview. Ann Periodontol 6:91-98

96. Preshaw PM (2008) Diabetes and periodontal disease. Int Dent J $58: 237-243$ 